\title{
Coupling of university photonic courses with clinical training
}

\section{S. V. Yakovleva}

S. V. Yakovleva, "Coupling of university photonic courses with clinical training," Proc. SPIE 9664, Ninth International Topical Meeting on Education and Training in Optics and Photonics, 96640H (24 October 2005); doi: $10.1117 / 12.2207781$

SPIE Event: Ninth International Topical Meeting on Education and Training in Optics and Photonics, 2005, Marseille, France 
This paper is freely available as a resource for the optics and photonics education community.

\title{
Ref ETOP096
}

\section{Coupling of university photonic courses with clinical training.}

\author{
S.V.Yakovleva
}

Ural State Technical University, 19 Peace Street, Ekaterinburg, Russia, 620002

\begin{abstract}
Biomedical engineering is a newly developed interdisciplinary subject developed from the combination of modern life science, medical science and engineering. Laser applications in biophysics and medicine plays one of the leading role in modern biomedical technology. But at the same time traditional photonic courses for biophysics include insufficient materials about biomedical laser applications. The current paper discuss the possibility of traditional courses enhancement by including laser demonstrations and training based on coupling of Technical university educational program and training program of Ekaterinburg Clinical center "Cardiology".
\end{abstract}

\section{Key words}

Biomedical engineering, medical science, engineering, laser applications in biophysics, biomedical technology, biomedical applications, educational program, training program.

\section{Summary}

It is impossible to reach a considerable progress in a modern medicine without creating medical technologies, where the latest achievements of fundamental analyses in the spheres of physics, chemistry, mathematics and biology are used. The latest years' achievements, which were get at the boundary of these sciences, provide a powerful potential for the development of the medical technology (equipment).

A new speciality has appeared at the Physico-technical faculty- engineers in medicine. It is based on a building of such knowledge on the board of different sciences and is aimed at coupling of different knowledge in a unique picture about the human's organism. This speciality is first of all directed on providing a qualified development of a technico- medical resource of medicine.

The purpose of this speciality is form the specialists of photonics, device building and biophysics, taking into consideration a medical specification.

The aspect of medicine is manyfunctional, as the object of the medical analyses- the human being - is very complicated. From the point of view of the accurate sciences the medicine is not rather formalized, it is more conservative.

The medical specification is introduced in this course of a new speciality by visiting the clinics, laboratories of the clinical center, participating in manipulations, headed by a doctor with a patient (operations, laser therapy). In addition, students get the sense of responsibility for the results of their deeds. Beyond the dull numbers of statistics the conscience of the socially valuable result appears. Taking part in various stages of examining and curing patients is one of the methods of determining their motivation while choosing a profession (an engineer on the medical equipment, a laboratory assistant making researching, a researcher of the fundamental proceses).

The medical technologies are complicated ones. They have all kinds of complicity (structural, organizational, algorithmic, systematic, cybernetic, and the complicity of choice) due to the complicity of an object and a high level of responsibility while making a solution of what technology to apply. A specialist who develops a technology should keep in mind all the technological chain, be conscious of all the aspects and be able to value his own place in it. 
Here is an example of a technological process of interaction of a laser radiation with a biological object. The process includes three aspects: treatment, diagnostics, and prognosis. A combination of a medical technology and the physics of the processes is the most effective thing for every aspect, which results in an excess system knowledge with new properties. The specialists in applying physical methods in medicine must get an integrated (composite) education, including the theoretical bases of the accurate sciences and a practice of clinical researches compulsory (obligatory).

An adoption of such experts into clinics result in an activation of the medical researching potential. As medics are conservatives, then for a development of medical technologies they need to cooperate with specialists in the sphere of techniques and natural sciences, who do not have any limits (conservative approach) in the educational course.

For all these reasons we were have to work out an educational program for students including a theoretical course (biophysics, photonics), practical studies in a laboratory on the bases of the biophysical laboratory of the University and studies on the basis of the leading scientific medical institution - the clinical center "Cardiology". There is a scientific institution of biophysics and an adoption of an optical radiation in a practical medicine.

The educational cycle of "the engineers in medicine" is carried out the following way. During the $1^{\text {st }}$ year students get acquainted with the scientific resources of the clinical center "Cardiology". The $2^{\text {nd }}$ year is devoted to studying the theoretical bases of the biophysics and biophotonics and students have practical studies in the university laboratory. At the $3^{\text {rd }}$ year they begin doing their research work, within the framework of which they get to know with the laboratory work of the clinical center "Cardiology" (the biochemical laboratory, the biophysical laboratory of a myocardial infarction, the biophotonics laboratory). In these laboratories students get practical knowledge about the interactive mechanisms of an optical radiation with an organism on an organismal level, and about the diagnostic methods of an effectiveness of a radiation application.

At the senior years on the basis of the two laboratories students study the influence of an optical radiation at the overmolecular level (a research of the structural peculiarities).

On the basis of the educational laboratories a theory was worked out. It enables to explain the interaction mechanisms of the laser radiation with a biological object and therefore to construct methods of diagnostics and treatment with a laser radiation.

It was experimentally found out that there are various mechanisms responsible for changes in the state of a biological system in the laser field with a low level of energy. "The trigger mechanism " of these changes, which can be seen through the interactive effect on the biological fluid, runs at low temperature of an electro- magnetic field and is of a global nature. The structural changes can be seen not only in the force place (place influenced), but also in all the organs and tissues or a biological object. Experimentally in was ascertained that the initial interactive mechanism of a laser radiation of low intensity with a biological object is a changing mechanism of a structure of diaphragms formation.

According to an offered physical model of a biological fluid even light structural changes lead to a diaphragm destabilization in general. In an electromagnetic field the reason for such a destabilization is a reorientation of the polar groups of a lipid biological layer of the diaphragm surface. Which through the connection lipids- proteins lead to a further influence on the cells' energy production, immune and fermented reactions.

The developed laser therapy system has its own structure and is added with new diagnostic methods on the basis of the knowledge about a physical mechanism of operating of a laser radiation on a biological object and a structurally modifying metastable model of biological fluid.

New criteria of effectiveness are designed and used. They are made on an integration of knowledge about the elementary interactions of low intensity laser irradiation with substance, a biological activity of the products of interaction, the biophysical transmutations, 
initiated by the products of interaction on the molecular, overmolecular, cellular, tissual and organic levels.

The specialists' training goes within the framework of an integrative medicine, providing medical, physico- mathematical, informationally cognitive, and systemo- technical aspects, what provides a complex interactive analysis of a laser radiation and a biological object, development of diagnostically technological, organizationally administrative and criterionally- valuable practice in patients' interests. 